Supporting Information for:

\title{
Non-Functionalized PNAs as Beacons for Nucleic Acids Detection in a Nanopore System
}

Alina Asandei ${ }^{1, \#,}$ Loredana Mereuta ${ }^{2, \#, ~ J o n g g w a n ~ P a r k ~}{ }^{3}$, Chang Ho Seo ${ }^{3}$, Yoonkyung Park ${ }^{4, *}$, Tudor Luchian ${ }^{2, *}$

${ }^{1}$ Interdisciplinary Research Institute, Sciences Department, 'Alexandru I. Cuza’ University, Iasi, Romania, 700506

${ }^{2}$ Department of Physics, 'Alexandru I. Cuza' University, Iasi, Romania, 700506

${ }^{3}$ Department of Bioinformatics, Kongju National University, Kongju, South Korea, 32588

${ }^{4}$ Department of Department of Biomedical Science and Research Center for Proteinaceous Materials (RCPM), Chosun University, Gwangju, South Korea, 61452

\# These authors contributed equally

*Corresponding authors (luchian@uaic.ro and y k park@,chosun.ac.kr) 
The primary sequences of the PNAs and ssDNAs implicated in this work are shown in Table 1, and were synthesized and purified by Panagene Inc., South Korea (PNA) and SigmaAldrich, Germany (ssDNAs). Potassium chloride $(\mathrm{KCl})$, sodium chloride $(\mathrm{NaCl})$, ultra-pure water (DNAase and RNAase free), $n$-pentane, hexadecane, EDTA, buffers (Tris and HEPES), DMSO and $\alpha$-hemolysin $(\alpha$-HL) were purchased from Sigma-Aldrich, Germany.

Table S1 Primary sequence of the PNA and ssDNA strands. The underlined region within the nDNA1_5 and nDNA7_5 represents the mismatches introduced to the complementary ssDNA (cDNA) relative to the PNA sequence.

\begin{tabular}{|c|c|c|}
\hline Polynucleotide & Primary sequence & Mw (g/mol) \\
\hline PNA & 5'-GTGATATACGGTGAT-3' & 4156.9 \\
\hline cDNA & 5'-ATCACCGTATATCAC-3' & 4499 \\
\hline nDNA1_5 & 5'-TTCACCGTATATCAC-3' & 4490 \\
\hline nDNA7_5 & 5'-TAGTGTTTATATCAC-3' & 4560 \\
\hline
\end{tabular}

All ssDNAs samples, obtained in dried form, were dissolved in $1 \mathrm{M} \mathrm{NaCl}$ solution in ultrapure water buffered with TE (10 mM Tris, $1 \mathrm{mM}$ EDTA) at $\mathrm{pH}=8.2$, to obtain stock solutions of $50 \mu \mathrm{M}$. A stock solution of $100 \mu \mathrm{M}$ PNA was prepared in the same buffer, and to enhances PNA solubility, small amounts $(\sim 6 \mu \mathrm{l})$ of DMSO were added. All samples were vigorously stirred using a Stuart vortex-mixer with BioCote (Sigma-Aldrich, Germany) at $1400 \mathrm{rpm}$ for 3 minutes each, in continuous mode. To improve rehydration the samples were heated up to $95{ }^{\circ} \mathrm{C}$ for $20 \mathrm{~min}$ using an IKA Digital Block Heater (Cole-Parmer, U.S.A.), and slowly cooled down to $22{ }^{\circ} \mathrm{C}$. This 
protocol was repeated before starting every experiment. All stock solutions were kept at $-20^{\circ} \mathrm{C}$ before and after use.

The electrophysiology experiments were undertaken in $3 \mathrm{M} \mathrm{KCl}$ electrolyte solution, buffered with $10 \mathrm{mM}$ HEPES at $\mathrm{pH}=7.2$ at a room temperature of $\sim 23{ }^{\circ} \mathrm{C}$. Single-molecule nanopore recordings were performed according to our previously described protocols ${ }^{1,2}$. In short, planar lipid membranes were made of 1,2-diphytanoyl-sn-glycerophosphocholine (Avanti Polar Lipids, Alabaster, AL, U.S.A.) and obtained using the Montal-Mueller technique ${ }^{2,3}$ across a 1:10 hexadecane/pentane (HPLC-grade, Sigma-Aldrich, Germany) pretreated, $\sim 120 \mu \mathrm{m}$ in diameter orifice punctured in a $25 \mu \mathrm{m}$ thick Teflon film (Goodfellow, Malvern, MA, U.S.A.) that separated the cis (grounded) and trans chambers of the recording cell. Having completed the mechanical stability check on the lipid bilayer, $\sim 0.5-2 \mu \mathrm{L}$ of wild-type $\alpha$-hemolysin ( $\alpha$-HL) (Sigma-Aldrich, Germany) were added from a monomeric stock solution made in $0.5 \mathrm{M} \mathrm{KCl}$ to the grounded, cis chamber under continuous stirring, for about 5-10 minutes. Once a single $\alpha$-HL heptamer spontaneously assembled in the lipid membrane, ssDNAs and PNA were added from the stock solution to the cis-side of the membrane. Measurements were carried out by applying transpositive voltages ranging between $+120 \div+200 \mathrm{mV}$. The resulting currents, reflecting the ssDNAand PNA-DNA duplexes - induced reversible current blockades of the $\alpha$-HL pore, were recorded via two $\mathrm{Ag} / \mathrm{AgCl}$ electrodes coupled with an Axopatch 200B (Molecular Devices, U.S.A.) set in the voltage-clamp mode. Raw data were low-pass filtered at $10 \mathrm{kHz}$ and then digitized with a NI PCI 6221, 16-bit acquisition board (National Instruments, U.S.A.) at a sampling frequency of 50 $\mathrm{kHz}$, with a custom-designed virtual instrument developed in LabVIEW 8.20 (National Instruments, U.S.A.). To avoid electromagnetic and mechanic interference, the bilayer chamber was housed in a Faraday cage (Warner Instruments, U.S.A. or Thorlabs, U.S.A.), and placed on 
the top of a vibration-free platform (Bench Mate 2210, Warner Instruments, U.S.A.). The statistical analysis of the stepwise reversible changes on the electric current through a single $\alpha$-HL protein, reflecting ssDNA- or PNA-DNA - induced blockades, were analyzed within the statistics of exponentially distributed events from data collected during multiple recordings, as previously described $^{1,2}$. The analysis on ionic current values through a single nanopore, to quantify relative blockade amplitudes induced by PNA-DNA duplexes or ssDNAs, were done by measuring individually the extent of such blockade events stemming from at least three experiments, and then performing the data analysis on pooled data. Data graphing and statistics were done with the help of the Origin 6 (OriginLab, Northampton, MA, U.S.A.) and pClamp 6.03 (Molecular Devices, U.S.A.) software.

1. Ciuca, A.; Asandei, A.; Schiopu, I.; Apetrei, A.; Mereuta, L.; Seo, C.-H.; Park, Y.; Luchian, T. Single Molecule, Real-Time Dissecting of Peptide Nucleic Acids-DNA Duplexes with a Protein Nanopore Tweezer. Anal. Chem. 2018, 90, 7682-7690.

2. Asandei, A.; Ciuca, A.; Apetrei, A.; Schiopu, I.; Mereuta, L.; Seo, C. H.; Park, Y.; Luchian, T. Nanoscale Investigation of Generation 1 PAMAM Dendrimers Interaction with a Protein Nanopore. Sci. Rep. 2017, 7, 6167, doi: 10.1038/s41598-017-06435-1.

3. Montal, M.; Mueller, P. Formation of Bimolecular Membranes from Lipid Monolayers and a Study of their Electrical Properties. Proc. Natl. Acad. Sci. U. S. A. 1972, 69, 3561-3566. 